\title{
REVOLUTION UND GESCHICHTSPHILOSOPHIE. ZUM BEGRIFF DER KRITIK BEIM FRÜHEN UND BEIM SPÄTEN MARX
}

\author{
Georg Spoo ${ }^{1}$
}

\section{Zusammenfassung:}

Der Beitrag widmet sich einem zentralen Dilemma radikaler Kritik: Entweder akzeptiert radikale Kritik den Maßstab des kritisierten Gegenstandes, verliert dadurch aber ihre Radikalität, oder ihre Radikalität gründet auf eigenen Maßstäben, dann bleiben sie dem kritisierten Gegenstand aber äußerlich. Der Beitrag rekonstruiert dieses Dilemma und mögliche Lösungen anhand von drei Modellen der Kritik in Marx' Früh- und Spätwerk. Dabei geht es nicht vorrangig um die Frage, was Marx an der bürgerlichen Gesellschaft kritisiert, sondern wie er sie kritisiert und welche Antworten er auf die Aporie radikaler Kritik bietet.

Schlagwörter: Kritik. Normativität. Revolution. Philosophie.

\section{REVOLUTION AND PHILOSOPHY OF HISTORY. THE CONCEPT OF CRITIQUE IN MARX' EARLY AND LATE WORK}

\begin{abstract}
:
The paper is devoted to a main dilemma of radical critique: Either radical critique accepts the criterion of the criticized object, but thereby loses its radicality, or its radicality is grounded on its own criterions, but remains external to the criticized object. This dilemma and possible solutions are reconstructed on the basis of three models of critique in Marx's early and late work. The paper is not primarily concerned with the question of what Marx criticizes about bourgeois society, but rather how he criticizes it and what answers he offers to the aporia of radical critique.
\end{abstract}

Keywords: Critique. Normativity. Revolution. Philosophy.

Die Philosophie von Karl Marx ist eine radikale Kritik der liberalen bürgerlichen Gesellschaft. Jede radikale Kritik ist mit einer grundlegenden Aporie konfrontiert: Wenn radikale Kritik an die normativen Maßstäbe ihres Gegenstandes anschließt, ist sie nicht länger radikal, da sie ihren Gegenstand an seinen eigenen Maßstäben misst und ihn dadurch affirmiert. Trägt sie jedoch eigene äußerliche Maßstäbe an den Gegenstand heran, um seine Affirmation zu vermeiden, verfehlt sie ihren Gegenstand, weil sie ihn an fremden Maßstäben misst. Die Radikalität und der Gegenstandsbezug von Kritik scheinen somit in einer virulenten Spannung zu stehen. Mindestens ebenso wichtig wie die umstrittene Frage, was Marx an der bürgerlichen Gesellschaft eigentlich genau kritisiert - ob Entfremdung, Ausbeutung oder Dysfunktionalität - ist

1 Georg Spoo ist wissenschaftlicher Mitarbeiter am Philosophischen Seminar der Albert-LudwigsUniversität Freiburg. Er arbeitet an einer Dissertation zur Kritik des Idealismus bei Kant und Fichte. Email: georg.spoo@philosophie.uni-freiburg.de 
daher die Frage, wie Marx die bürgerliche Gesellschaft kritisiert; und ob es ihm gelingt eine nicht-aporetische radikale Kritik zu begründen.

Diesen Fragen soll im Folgenden nachgegangen werden, indem anhand seines Frühund Spätwerks, vor allem der Doktordissertation, der Judenfrage und dem Kapital, drei verschiedene Kritikmodelle rekonstruiert werden: das linkshegelianische Modell (1.), nach Marx' sozialistischer Wende (2.) das sozialrevolutionäre Modell (3.) und schließlich das geschichtsphilosophische Modell (4.). Diese drei Modelle geben unterschiedliche Antworten auf die Frage, wie radikale Kritik möglich ist und sind mit unterschiedlichen politischen Konsequenzen verbunden. Alle drei Modelle haben Stärken und Schwächen. Deshalb erscheint es für die Kritik der Gegenwart sinnvoll, diese Modelle nicht als Alternativen zu verstehen, sondern ineinander zu integrieren (5.).

\section{Linkshegelianische Kritik}

Das erste Kritikmodell entwickelt Marx im Zeitraum zwischen 1840 und 1842 in seiner Doktordissertation über die Differenz der demokritischen und epikureischen Naturphilosophie (1840/1841) sowie seinen frühesten Zeitungsartikeln aus dem Jahr 1842, in denen er emphatisch die Pressefreiheit gegen die Zensurbestrebungen des preußischen Staates verteidigt. Als Sozialist oder Kommunist versteht sich Marx zu dieser Zeit ausdrücklich nicht (vgl. MEW 1, 105-108), sondern er argumentiert eher radikal liberal und demokratisch. Sein Kritikbegriff ist zumindest rhetorisch stark durch Hegel beeinflusst, weicht in entscheidenden Aspekten aber von Hegel ab. Das in dieser Zeit entwickelte Kritikmodell lässt sich als linkshegelianisch bezeichnen.

In diesem Modell bedeutet Kritik, das, was an der Wirklichkeit nur scheinhaft und unvernünftig ist, von einem philosophischen „Standpunkt der Idee“ (MEW 1, 50) anhand eines Maßstabes der Wahrheit und der Vernunft zu kritisieren. Das, was nur vernünftig zu sein scheint, muss kritisiert werden, um das wirkliche Wesen der Dinge erkennen zu können. Die Kritik, so Marx in seinen Debatten über Pressefreiheit, verlässt hierfür den Standpunkt, „der mir die Welt und die menschlichen Verhältnisse

\begin{tabular}{|l|l|l|l|l|}
\hline Q Povista Qialectus & Ano 9 & n. 18 & Outubro 2020 & p. 202-218 \\
\hline
\end{tabular}


nur in ihrem äußerlichen Schein zeigt, ihn als unfähig zu erkennen, den Wert der Dinge zu beurteilen“ (MEW 1, 50). In starker Anlehnung an Hegelsche Terminologie schreibt Marx weiterhin: „Wir müssen also das Maß des Wesens der innern Idee an die Existenz der Dinge legen“ (MEW 1, 50). In dieser Prüfung, ob die bloße Existenz der Dinge dem normativen Anspruch der Idee dieser Dinge entspricht, liegt für Marx das Wesen der Kritik. In seiner Doktordissertation schreibt er: „Es ist die Kritik, die die einzelne Existenz am Wesen, die besondere Wirklichkeit an der Idee mißt"“ (MEW 40, 326/328) Kritik bedeutet demnach also, vereinfacht gesagt, die schlechte und falsche Wirklichkeit an der guten und richtigen Idee dieser Wirklichkeit als Maßstab zu messen.

Obwohl Marx diese Auffassung in auffälliger terminologischer Nähe zu Hegel formuliert, distanziert er sich mit ihr sachlich von Hegel. Hegel fasst die Idee bzw. die Vernunft nämlich nicht bloß als ein besonderes Prinzip der Philosophie, sondern vertritt die Auffassung, dass die menschliche Wirklichkeit, zumindest grundsätzlich, immer schon auf eine rationale Weise strukturiert ist. Die Idee bzw. die Vernunft ist der Wirklichkeit daher nicht entgegengesetzt, sondern mit ihr identisch. Das heißt allerdings auch für Hegel nicht, dass alles, was ist, automatisch vernünftig ist. Das, was nicht vernünftig, sondern bloß zufällig und unwesentlich ist, bezeichnet Hegel als bloße ,Existenz', die normativ unterhalb der vernünftig strukturierten ,Wirklichkeit rangiert. Die Existenz dafür zu kritisieren, dass sie nicht mit der Vernunft übereinstimmt, ist demnach eine Tautologie. Für eine Kritik, die die Existenz an der Idee misst, hat Hegel folglich kaum mehr als Spott übrig. In der Enzyklopädie schreibt er:

[W]er wäre nicht so klug, um in seiner Umgebung vieles zu sehen, was in der Tat nicht so ist, wie es sein soll? Aber diese Klugheit hat unrecht, sich einzubilden, mit solchen Gegenständen und deren Sollen sich innerhalb der Interessen der philosophischen Wissenschaft $\mathrm{zu}$ befinden. Diese hat es nur mit der Idee $\mathrm{zu}$ tun, welche nicht so ohnmächtig ist, um nur zu sollen und nicht wirklich zu sein. (Hegel 1830, 49)

Zwar ist der von Marx vertretene Kritikbegriff etwas weniger harmlos, als die von Hegel verspottete ,kritische Klugheit‘. Denn Marx knüpft an der Differenz zwischen Wirklichkeit und Existenz bei Hegel an und weitet sie gegen Hegel zur Differenz zwischen Idee und Wirklichkeit aus, um auch die Wirklichkeit durch eine von ihr

\begin{tabular}{|l|l|l|l|l|}
\hline Q Povista Qialectus & Ano 9 & n. 18 & Outubro 2020 & p. 202-218 \\
\hline
\end{tabular}


unterschiedene Vernunft kritisieren zu können, die Hegel noch mit der Wirklichkeit identifiziert hatte. Wenn aber nicht nur die Existenz, d.h. letztlich historisch zufällige und strukturell bedeutungslose Aspekte der Wirklichkeit, sondern auch die Wirklichkeit selbst, d.h. ihre Grundstrukturen, nicht mit der Idee übereinstimmt, hört die Idee auf, Element der Wirklichkeit zu sein. Damit wird offenbar die gegenüber Kant fortschrittliche Position Hegels aufgegeben, dass Vernunft nicht nur subjektiv und theoretisch als geistige Fähigkeit von Individuen oder als partikulares Prinzip der Philosophie verstanden werden darf, sondern sie sich auch praktisch in objektiven und gesellschaftlichen Strukturen manifestiert. Die Idee wird bei Marx zu einem normativen Prinzip der subjektiven Vernunft, das der unvernünftigen objektiven gesellschaftlichen Wirklichkeit entgegengehalten wird. ${ }^{2}$ Das hat auch politische Konsequenzen: Die Kritik an der Wirklichkeit auf Grundlage der Idee scheint im linkshegelianischen Modell vorrangig eine Aufgabe der von der Praxis noch losgelösten Theorie (vgl. MEW 40, 327), von kritischen Intellektuellen oder philosophisch gebildeten Journalisten, wie Marx zu dieser Zeit selbst einer ist, d.h. der bürgerlichen Öffentlichkeit.

Marx' Abgrenzung von Hegel eröffnet eine grundsätzliche systematische Aporie radikaler Kritik: Die Hegelsche Identifikation von Vernunft bzw. Idee und Wirklichkeit schließt die Möglichkeit radikaler Kritik der Wirklichkeit eigentlich aus. Denn in einer von Vernunft durchdrungenen Wirklichkeit kann es eigentlich kein rationales oder normatives Desiderat geben, das in dieser Wirklichkeit grundsätzlich verfehlt wird. Wenn eine radikale Kritik der Wirklichkeit möglich sein soll, müssen Wirklichkeit und Vernunft deshalb - wie bei Marx - voneinander getrennt werden. Mit dieser Trennung geht aber die Gefahr einher, Vernunft und Kritik als gegenüber der Wirklichkeit externe Ansprüche eines nur theoretischen und subjektiven Standpunktes zu verstehen. Die normativen Ambitionen der Kritik kommen dann über Sollensforderungen nicht hinaus. Sie bleiben ihrem Gegenstand, den praktischen und objektiven gesellschaftlichen Verhältnissen, äußerlich oder gehen gar an ihnen vorbei, und steuern auf einen vor- oder sogar unpolitischen Moralismus zu. Man könnte

2 Allerdings vertritt Marx diesen Kritikbegriff nicht naiv. In seiner Doktordissertation weist er darauf hin, dass eine Trennung der Idee von der Wirklichkeit nicht nur für die kritisierte Wirklichkeit, sondern auch für die Idee als Maßstab der Kritik problematisch ist (vgl. MEW 40, 329). Für sein erstes Kritikmodell bleibt diese Einsicht aber noch ohne Konsequenzen. Sie macht sich erst in den folgenden Modellen bemerkbar.

\begin{tabular}{|l|l|l|l|l|}
\hline Qevista Dialectus & Ano 9 & n. 18 & Outubro 2020 & p. 202-218 \\
\hline
\end{tabular}


dieses Problem als normativen Externalismus radikaler Kritik bezeichnen. Um dem Externalismus zu begegnen, ohne aber die Möglichkeit radikaler Kritik aufzugeben, müsste die Trennung von Vernunft und Wirklichkeit, anstatt als Trennung der Philosophie von der gesellschaftlichen Wirklichkeit, vielmehr innerhalb der Wirklichkeit selbst verortet werden. Erst wenn das gelingt, könnte Marx einerseits an der entscheidenden Hegelschen Einsicht festhalten, dass die Vernunft (sowie die Unvernunft) nie bloß individuell oder vorgesellschaftlich, sondern immer auch Teil der gesellschaftlichen Wirklichkeit ist - ohne sich damit aber andererseits zugleich auf Hegels vollständige Identifizierung von Vernunft und Wirklichkeit verpflichten zu müssen und damit wiederum die Möglichkeit radikaler Kritik preiszugeben. Dieser Herausforderung stellt sich Marx in der 1843 verfassten Kritik des Hegelschen Staatsrechts und in dem ebenfalls 1843 verfassten und 1848 in den DeutschFranzösischen Jahrbüchern erschienenen Text Zur Judenfrage. Mit seiner in diesen Texten radikalisierten Kritik der bürgerlichen Gesellschaft (2.) radikalisiert Marx auch seinen Kritikbegriff (3. u. 4.).

\section{Kritik der bürgerlichen Gesellschaft}

Der Text Zur Judenfrage kann als Marx' erstes Programm radikaler Gesellschaftskritik gelesen werden: In diesem Text kritisiert er nicht mehr nur die politische, sondern auch die ökonomische Wirklichkeit der bürgerlichen Gesellschaft, die er schon mit seinem Ende 1842 verfassten Beitrag zu den Debatten über das Holzdiebstahlsgesetz im Rheinischen Landtag und seinen Untersuchungen über die soziale Lage der Moselbauern 1843 (vgl. MEW 1, 172-199) in den Blick genommen hatte. Die gesellschaftstheoretischen Ansätze zu einer Kritik der bürgerlichen Gesellschaft in der Kritik des Hegelschen Staatsrechts werden in der Ende 1843 verfassten Schrift Zur Judenfrage zu einer eigenständigen kritischen Gesellschaftsanalyse ausgebaut. In den 1844 verfassten Kritischen Randglossen bekennt sich Marx vor diesem Hintergrund dann ausdrücklich zum Sozialismus und bricht mit seinen zuvor eher radikal liberalen Ansichten. Mit dieser Radikalisierung

\begin{tabular}{|l|l|l|l|l|}
\hline Q Rovista Dialectus & Ano 9 & n. 18 & Outubro 2020 & p. 202-218 \\
\hline
\end{tabular}


transformiert er das linkshegelianische Kritikmodell in ein sozialrevolutionäres Kritikmodell. $^{3}$

Im Staat, so Marx in der Judenfrage, führt der Mensch als „Gemeinwesen“ sein „Gattungsleben [...] im Gegensatz zu seinem materiellen Leben.“ (MEW 1, 354-355). In ihm gilt er als „souveränes, als höchstes Wesen“ (MEW 1, 360). In der bürgerlichen Gesellschaft bzw. auf dem kapitalistischen Markt hingegen lebt der Mensch als „Privatmensch“ „,egoistisch[...]“, als ein ,auf sein Privatinteresse und seine Privatwillkür zurückgezogenes und vom Gemeinwesen abgesondertes Individuum“ (MEW 1, 366), das andere Menschen „als Mittel betrachtet, sich selbst zum Mittel herabwürdigt und zum Spielball fremder Mächte wird“ (MEW 1, 355). In ihr lebt der Mensch, „wie er durch die ganze Organisation unserer Gesellschaft verdorben, sich selbst verloren, veräußert, unter die Herrschaft unmenschlicher Verhältnisse und Elemente gegeben ist" (MEW 1, 360). In der politischen Sphäre des Staates, so lässt sich Marx’ Diagnose zusammenfassen, ist der Mensch demnach ein allgemeines Gattungs- und Gemeinwesen, in der ökonomischen Sphäre der bürgerlichen Gesellschaft hingegen ist er ausschließlich ein vereinzeltes Individuum. Als homo politicus verfügt der Mensch über Selbstbestimmung und ist der Zweck politischen Handelns. Als homo oeconomicus hingegen ist der Mensch fremdbestimmt und nur Mittel für andere Zwecke.

Entscheidend für Marx' Weiterentwicklung seiner bisherigen Position ist, dass er die politische Sphäre des Staates mit der Idee und die ökonomische Sphäre der bürgerlichen Gesellschaft mit der Existenz identifiziert und sein hegelianisches Vokabular dadurch gesellschaftstheoretisch konkretisiert: In den Kritischen Randglossen, die er wenige Monate nach der Judenfrage verfasst hat, bezeichnet er den Gegensatz zwischen dem Staat und der bürgerlichen Gesellschaft als den „Gegensatz zwischen der allgemeinen Idee und der individuellen Existenz des Menschen“ (MEW 1, 408; vgl. auch MEW 1, 360). Das ist gegenüber seinem früherem Kritikprogramm ein entscheidender Fortschritt: Die zunächst mit Hegel vorgenommene Unterscheidung zwischen der Idee bzw. der Vernunft und der bloßen Existenz, die Marx dann gegen Hegel zum Unterschied zwischen Idee und

3 Vgl. überblicksartig zum ,sozialrevolutionären' Marx mit Fokus auf den Begriff der Kritik u.a. Röttgers 1975, 253-277, Heinrich 2014, S. 86-120, Arndt 2012, S. 14-62 u. Strehle 2019.

\begin{tabular}{|l|l|l|l|l|}
\hline Revista Dialectus & Ano 9 & n. 18 & Outubro 2020 & p. 202-218 \\
\hline
\end{tabular}


Wirklichkeit ausgeweitet hatte, geht nicht mehr mit einer Trennung von subjektiven theoretischen oder normativen Vorstellungen einerseits und der objektiven praktischen Realität andererseits einher. Marx versteht Normativität jetzt nämlich nicht mehr bloß als subjektive Vorstellung, sondern in erster Linie als eine Normativität, die sich in gesellschaftlichen Institutionen ausdrückt. Der Gegensatz zwischen der Idee und der Wirklichkeit wird folglich nicht mehr als Gegensatz zwischen Idee und Wirklichkeit, sondern vielmehr als Gegensatz zwischen Staat und bürgerlicher Gesellschaft, d.h. als Gegensatz der gesellschaftlichen Wirklichkeit selbst verstanden. ${ }^{4}$ Damit verbindet Marx die Einsicht, dass die nicht-wirkliche Vernunft in der Form des Staates ebenso defizitär ist, wie die nicht-vernünftige Wirklichkeit in der Form der kapitalistischen Ökonomie: „Der wirkliche Mensch ist erst in der Gestalt des egoistischen Individuums, der wahre Mensch erst in der Gestalt des abstrakten citoyen anerkannt" (MEW 1, 370, erste Herv. G.S.). Wahrheit und Idee bleiben in der politischen Sphäre demnach unwirklich und abstrakt, weil sie auf einen gesellschaftlichen Sonderbereich beschränkt bleiben. Sie durchdringen nicht den größeren wirklichen Bereich der bürgerlichen Gesellschaft, nämlich die kapitalistische Ökonomie, die nicht die Vernunft in sich realisiert. Das Vernünftige bzw. Wahre bleibt partikular und ist nicht das Wirkliche und das allgemeine Wirkliche ist nicht das Wahre bzw. Vernünftige. Dieser Chiasmus formuliert die innere Selbstverkehrung und Selbstverfehlung der gesellschaftlichen Wirklichkeit: Die bürgerliche Gesellschaft ist eine „verkehrte Welt“ (MEW 1, 340). Das Ziel radikaler Kritik kann demnach nicht mehr darin bestehen, die selbst einseitige und defizitäre Idee zum Maßstab der defizitären Wirklichkeit zu machen, sondern darin, die Trennung und Entgegensetzung beider in ihrer Gestalt von Staat und Gesellschaft aufzuheben.

\section{Sozialevolutionäre Kritik}

Dementsprechend übt Marx eine deutliche (Selbst-)Kritik an seinem früheren Kritikmodell. Das Modell der ,linkshegelianischen“ Kritik bezeichnet er jetzt polemisch als ,vulgär‘ oder ,dogmatisch“ (vgl. MEW 1, 296). Diese Kritik „findet überall Widersprüche. Das ist selbst noch dogmatische Kritik, die mit ihrem

4 Heinrich 2014, S. 103 ist hingegen der Auffassung, dass sich die grundlegende Struktur der Marx'schen Konzeption in der Judenfrage nicht geändert habe.

\begin{tabular}{|l|c|c|c|c|}
\hline Rovista Dialectus & Ano 9 & n. 18 & Outubro 2020 & p. 202-218 \\
\hline
\end{tabular}


Gegenstand kämpft, so wie man früher etwa das Dogma der heiligen Dreieinigkeit durch den Widerspruch von eins und drei beseitigte." (MEW 1, 296) Diese Kritik, so lässt sich Marx Gedanke ausführen, hat ihren Gegenstand noch nicht zureichend verstanden und befindet sich in nur einer internen Auseinandersetzung mit ihm, da sie ihn als widersprüchlich kritisiert und für eine Seite dieses Widerspruches Partei ergreift. Dadurch bleibt sie dem Widerspruch aber verhaftet und reproduziert ihn. Sie ist nicht in der Lage, den Widerspruch ihres Gegenstandes aus seiner inneren Struktur zu erklären, ihn als hinsichtlich des Gegenstandes notwendig einzusehen, und damit auch eine normativ über den Gegenstand hinausführende Perspektive einzunehmen.

In genau solch einer Analyse der notwendigen Widersprüche der gesellschaftlichen Wirklichkeit besteht für Marx' das Wesen der sozialrevolutionären bzw., in Marx Worten, ,wahren' Kritik:

Die wahre Kritik dagegen zeigt die innere Genesis der heiligen Dreieinigkeit im menschlichen Gehirn. Sie beschreibt ihren Geburtsakt. So weist die wahrhaft philosophische Kritik der jetzigen Staatsverfassung nicht nur Widersprüche als bestehend auf, sie erklärt sie, sie begreift ihre Genesis, ihre Notwendigkeit. Sie faßt sie in ihrer eigentümlichen Bedeutung [...]. (MEW 1, 296)

Dieser Kritik kann es nicht darum gehen, nur einen Widerspruch zwischen der gesellschaftlichen Wirklichkeit und den gesellschaftlichen Normen zu konstatieren. Vielmehr muss dieser Widerspruch aus der inneren Struktur der bürgerlichen Gesellschaft selbst erklärt werden. Diesen Gedanken einer immanenten Kritik der gesellschaftlichen Wirklichkeit hat Marx in dem berühmten Diktum formuliert, „daß wir nicht dogmatisch die Welt antizipieren, sondern erst aus der Kritik der alten Welt die neue finden wollen“ (MEW 1, 344). Damit weist er die Trennung zwischen Normativität und Faktizität, d.h. die Trennung zwischen der Idee als dem Maßstab der Kritik und der kritisierten Wirklichkeit zurück, die noch das linkshegelianische Kritikmodell kennzeichnete. Zwar vermeidet das sozialrevolutionäre Kritikmodell dadurch das Problem des normativen Externalismus, ist allerdings mit zwei neuen Problemen konfrontiert. Das erste Problem könnte man als Problem der normativen Begründung bezeichnen: ${ }^{5}$ Wenn, wie in der von Marx intendierten immanenten Kritik, das, was sein soll, aus dem, was ist, entwickelt wird, handelt sich Marx dann

5 Vgl. zum Begründungsproblem auch Spoo 2020.

\begin{tabular}{|l|c|c|c|c|}
\hline Q & Onisita & Outubro 2020 & p. 202-218 \\
\hline
\end{tabular}


nicht einen naturalistischen Fehlschluss ein, der Faktizität mit Normativität verwechselt? Die Antwort auf diese Frage lautet, dass in Marx' an Hegel orientiertem Verständnis von Wirklichkeit Faktizität und Normativität keine Gegensätze, sondern gleichermaßen Aspekte der Wirklichkeit sind: Die gesellschaftliche Wirklichkeit weist als solche immer schon normative Aspekte und Dimensionen auf, die sich in gesellschaftlichen Praxen, Institutionen, Verhältnissen und Strukturen materialisieren. Eben darin besteht die Pointe von Marx' Gesellschaftstheorie in der Judenfrage, die Idee nicht philosophisch, sondern, in der Gestalt des Staates, als Teil der praktischen gesellschaftlichen Wirklichkeit zu verstehen. Daraus folgt, dass die radikale Kritik nicht bloß einseitig an die normativen Instanzen der gesellschaftlichen Wirklichkeit anschließt, um dann anzuprangern, dass sie permanent verletzt werden und ihre Achtung einzufordern. Vielmehr geht es Marx darum, zu zeigen, dass sich Normativität und Faktizität in der liberal-kapitalistischen Gesellschaft wechselseitig durcheinander hervorbringen und durchdringen: Sowohl die Hervorbringung als auch die Verletzung ihrer eigenen Normen ist in der Struktur der kapitalistischen Gesellschaft selbst immer schon gleichursprünglich angelegt - und deshalb ist eine radikale Veränderung dieser Gesellschaft nötig. Auch wenn sich das Problem der Begründung der Kritik so lösen lässt, stellt sich dem sozialrevolutionären Modell aber noch ein Anschlussproblem, das sich nicht so leicht innerhalb dieses Modells lösen lässt. Man könnte dieses zweite Problem als Problem des normativen Internalismus bezeichnen: Wie lässt sich aus der ,internen“ Diagnose, dass ein Gegenstand notwendigerweise selbstwidersprüchlich verfasst ist, der Schritt hin $\mathrm{zu}$ seiner radikalen Veränderung ableiten? Eine notwendige Selbstwidersprüchlichkeit, ja selbst eine notwendige Selbstverfehlung, führt nur dann zu einer Veränderung, wenn die Widerspruchsvermeidung oder Selbsterfüllung als normatives Ziel bereits vorausgesetzt sind. Es ist aber fraglich, ob diese normativen Voraussetzungen allein aus der internen Analyse der Widersprüche des Gegenstandes entwickelt werden können, oder ob sie nicht doch extern hinzutreten müssen, um eine Veränderung zu initiieren. In Marx sozialrevolutionärem Modell bleibt diese Frage jedenfalls ungelöst. Sie wird erst im geschichtsphilosophischen Kritikmodell aus dem Kapital beantwortet.

\begin{tabular}{|l|l|l|l|l|}
\hline Q Rovista Dialectus & Ano 9 & n. 18 & Outubro 2020 & p. 202-218 \\
\hline
\end{tabular}


Wie schon der linkshegelianische so hat auch der sozialrevolutionäre Kritikbegriff bestimmte politische Konsequenzen. Er ist mit der ihm von vornherein eingeschriebenen Perspektive einer Überwindung der gesellschaftlichen Verkehrungen und Widersprüche immer auch Element verändernder Praxis. Dieser praktische Impetus unterscheidet das sozialrevolutionäre Kritikmodell von dem rein theoretischen Status der Kritik im linkshegelianischen Modell. Der Ort der Kritik ist daher auch nicht mehr, wie noch in Marx' frühesten Schriften, der Diskurs bürgerlicher Öffentlichkeit, sondern er besteht in den gesellschaftlichen Kämpfen und Konflikten, in denen die gesellschaftlichen Widersprüche praktisch ausgetragen und auf die Spitze getrieben werden. Dieser radikale Begriff der Kritik ist somit selbst eine Kritik des liberal-bürgerlichen Verständnisses. Marx pointiert die politischen Konsequenzen seiner radikalen Kritikauffassung so, dass es darum geht, „unsre Kritik an die Kritik der Politik, an die Parteinahme in der Politik, also an wirkliche Kämpfe anzuknüpfen und mit ihnen zu identifizieren“ (MEW 1, 345). Der Gedanke dieser Parteinahme der Kritik wiederum lässt sich als eine Art Antwort auf das Problem des Internalismus interpretieren: Die Parteinahme versteht Marx nämlich als „Selbstverständigung (kritische Philosophie) der Zeit über ihre Kämpfe und Wünsche“" (MEW 1, 346). Marx ist der Auffassung, dass die Kämpfe und Konflikte entlang der Linien der gesellschaftlichen Widersprüche noch nicht von selbst zu einer gesellschaftlichen Transformation führen. Um das radikale Veränderungspotential der gesellschaftlichen Widersprüche wirklich zu aktivieren, bedarf es deshalb der sozialrevolutionären Kritik, die den sozialen Konflikten und Kämpfen ihren für sie zunächst nur impliziten radikalen und sozialrevolutionären Telos expliziert:

Die Reform des Bewußtseins besteht nur darin, daß man die Welt ihr Bewußtsein innewerden läßt, daß man sie aus dem Traum über sich selbst aufweckt, daß man ihre eignen Aktionen ihr erklärt. [...]. Es wird sich dann zeigen, daß die Welt längst den Traum von einer Sache besitzt, von der sie nur das Bewußtsein besitzen muß, um sie wirklich zu besitzen. (MEW 1, 346)

Im geschichtsphilosophischen Modell der Kritik bietet Marx eine andere Lösung für das Problem des normativen Internalismus an und mit dieser Lösung entfällt auch die bewusstseinsbildende und praktische Funktion der Kritik.

\begin{tabular}{|l|l|l|l|l|}
\hline Q Rovista Dialectus & Ano 9 & n. 18 & Outubro 2020 & p. 202-218 \\
\hline
\end{tabular}




\section{Geschichtsphilosophische Kritik}

Mit dem geschichtsphilosophischen Modell der Kritik im Kapital reagiert Marx nicht nur auf den normativen Externalismus des linkshegelianischen Modells, sondern ebenfalls auf den normativen Internalismus des sozialrevolutionären Modells. Seine geschichtsphilosophische Konzeption einer objektiven Selbstkritik und Selbstüberwindung der kapitalistischen Gesellschaft drängt dabei jedoch die politische und praktische Bedeutung von Kritik zurück. Im Kapital finden sich zwar kaum explizite Überlegungen zum Kritikbegriff, allerdings lässt sich aus der kapitalismuskritischen Gesamtargumentation des Werks und verstreuten Bemerkungen ein recht schlüssiges Kritikmodell rekonstruieren. ${ }^{6}$

Im ersten Abschnitt des Kapitals untersucht Marx die elementaren Strukturen kapitalistischer Gesellschaften, den Wert, die Ware und den Warentausch. Sie bilden die Grundlage für die Produktion- und Akkumulation von Kapital, weil die spezifisch kapitalistische Ausbeutung der Arbeitskraft nur möglich ist, wenn die Arbeit als Ware gekauft werden kann. Die Zirkulationssphäre des Marktes, in der die Marktteilnehmer mit freiem Willen einen Äquivalententausch eingehen, versteht Marx dabei - anders als man auf den ersten Blick meinen könnte - tatsächlich unpolemisch als eine Art Ursprungsort der bürgerlichen Ideale von Freiheit und Gleichheit:

Die Sphäre der Zirkulation oder des Warenaustausches, innerhalb deren Schranken Kauf und Verkauf der Arbeitskraft sich bewegt, war in der Tat ein wahres Eden der angebornen Menschenrechte. Was allein hier herrscht, ist Freiheit, Gleichheit, Eigentum und Bentham. (MEW 23, 189)

Nach seiner Untersuchung der Zirkulationssphäre analysiert Marx die Produktionssphäre und den Akkumulationsprozess des Kapitals und zeigt, dass der Kapitalismus notwendigerweise und aus innerer Konsequenz die rücksichtslose Ausbeutung von Mensch und Natur betreibt. Bevor Marx den Gesamtprozess kapitalistischer Akkumulation resümiert, hält er fest:

6 Vgl. u.a. zum Kritikbegriff im Kapital knapp Heinrich 2009.

\begin{tabular}{|l|l|l|l|l|}
\hline Q Ronista Dialectus & Ano 9 & n. 18 & Outubro 2020 & p. 202-218 \\
\hline
\end{tabular}


Sosehr die kapitalistische Aneignungsweise also den ursprünglichen Gesetzen der Warenproduktion ins Gesicht zu schlagen scheint, so entspringt sie doch keineswegs aus der Verletzung, sondern im Gegenteil aus der Anwendung dieser Gesetze. (MEW 23, 610)

Freiheit und Gleichheit als Ideale der bürgerlichen Gesellschaft widersprechen demnach nicht der Ungleichheit und Unfreiheit im Kapitalismus, sondern bringen diese systematisch hervor. Nicht die Verletzung der Ideale der bürgerlichen Gesellschaft, so Marx' im Kapital radikalisierte Kritik, sondern ihre Realisierung führt zur Ausbeutung der Lohnabhängigen. Das Kernproblem ist daher nicht mehr, anders als noch in der Judenfrage, dass die bürgerliche Gesellschaft aus innerer Notwendigkeit ihre Ideale verletzt, sondern es sind diese Ideale selbst, die zu Ausbeutung und Herrschaft führen.

Vor diesem Hintergrund verschärft Marx auch nochmal seine Kritik der linkshegelianischen Kritik und des normativen Externalismus. Er wirft dieser Kritik jetzt nicht mehr, wie noch zur Zeit der Judenfrage, vor, dass sie hilflos mit ihrem Gegenstand ringt, weil sie ihn trotz aller genuin kritischen Absicht nicht zureichend verstanden hat. Mit dieser früheren Diagnose konzediert Marx einer solchen Kritik immerhin noch eine echte kritische Spannung zu ihrem Gegenstand. Im Kapital spricht er ihr eine solche Spannung vollends ab. Marx' entsprechende Argumentation lässt sich anhand von zwei Optionen wie folgt zuspitzen: Entweder trifft die Beurteilung der Wirklichkeit anhand ihrer Idee diese Wirklichkeit. Dann ist sie aber letztlich keine Kritik mehr, sondern eine Bejahung, weil sie bestenfalls die strukturellen und funktionalen Normen dieser Wirklichkeit einklagt. Oder sie zielt darauf $\mathrm{ab}$, als wirkliche Kritik, andere Normen einzuklagen, dann misst sie die Wirklichkeit aber an einem ihr fremden Maßstab und geht schlichtweg an ihr vorbei. Als Vertreter der ersten Option, d.h. einer immanenten (Schein-)Kritik der bürgerlichen Gesellschaft, nennt Marx den „Freihändler vulgaris“, der der Zirkulationssphäre seine „Anschauungen, Begriffe und Maßstab für sein Urteil über die Gesellschaft des Kapitals und der Lohnarbeit entlehnt“ (MEW 23, 191). Die im Warentausch geltenden Ideen von Freiheit und Gleichheit als die urtypischen Ideen der kapitalistischen Produktionsweise sind aber nicht geeignet, die auf den Warentausch gegründete kapitalistische Produktions- und Akkumulationsweise zu kritisieren, sondern sie bejahen ihn vielmehr. Als Vertreter der zweiten Option, d.h.

\begin{tabular}{|l|l|l|l|l|}
\hline Q Povista Dialectus & Ano 9 & n. 18 & Outubro 2020 & p. 202-218 \\
\hline
\end{tabular}


einer Kritik anhand äußerlicher Maßstäbe, nennt Marx paradigmatisch Proudhon und Gilbart, die den Kapitalismus auf naturrechtlicher Grundlage kritisieren:

Mit Gilbart [...] von natürlicher Gerechtigkeit hier zu reden ist Unsinn. Die Gerechtigkeit der Transaktionen, die zwischen den Produktionsagenten vorgehn, beruht darauf, daß diese Transaktionen aus dem Produktionsverhältnissen als natürliche Konsequenz entspringen. (MEW 25, 351f.)

Und gegen Proudhon gerichtet schreibt Marx:

Weiß man etwa mehr über den „Wucher“, wenn man sagt, er widerspreche der ,justice éternelle“ und der „équité éternelle“ und der „mutalité éternelle“ und anderen „vérités éternelles“, als die Kirchenväter wußten, wenn sie sagten, er widerspreche der „grâce éternelle“, oder „foi éternelle“, der „volonté éternelle de dieu“? (MEW 23, 100)

In dieser Polemik gegen Gilbart und Proudhon, die die Wirklichkeit anhand eines postulierten Ideals kritisieren, eignet Marx sich gewissermaßen den Spott Hegels gegen eine Kritik des abstrakten Sollens an, die noch Marx linkshegelianisches Kritikmodell getroffen hatte: eine bloße Kritik des Kapitalismus anhand von naturrechtlich oder anderweitig extern begründeten Normen versteht ihn nicht, trifft ihn nicht und ist ihm deshalb hilflos ausgeliefert.

Es ist keine Überraschung, dass sich Marx nicht nur in der Kritik eines falschen Kritikverständnisses, sondern auch in der Konzeption des richtigen Kritikmodells Hegel wieder annähert. In der Vorrede zur Phänomenologie des Geistes schreibt Hegel: „Das leichteste ist, was Gehalt und Gediegenheit hat, zu beurteilen, schwerer, es zu fassen, das schwerste, was beides vereinigt, seine Darstellung hervorzubringen.“ (Hegel 1807, 11). Hier formuliert Hegel programmatisch die Idee, das Verstehen des Gegenstandes und dessen Kritik zu verbinden, was er dann methodisch sowohl in der Phänomenologie als auch in der Logik durchführt: Die Kritik ist demnach keine Beurteilung der Sache von einem der Sache äußerlichen Standpunkt. Eine solche Kritik, die die Sache nur beurteilt, ohne sie zu ,fassen', hat mit der Sache letztlich eigentlich gar nichts zu tun und schlägt deshalb als Kritik dieser Sache fehl. Das Verstehen der Sache hingegen, das auf Kritik der Sache verzichtet, verkennt die innere Dynamik und die innere Spannung einer Sache und fasst sie deshalb nur verkürzt und einseitig. In der gelungenen Darstellung einer Sache verbinden sich beide Hinsichten: Kritik bedeutet hier nicht, von außen eine Kritik der Sache zu üben,

\begin{tabular}{|l|l|l|l|l|}
\hline Q Povista Dialectus & Ano 9 & n. 18 & Outubro 2020 & p. 202-218 \\
\hline
\end{tabular}


sondern darzustellen, wie sich die Sache gewissermaßen selbst kritisiert, d.h., allgemein formuliert, ihre Ambivalenzen, ihre Spannungen und Widersprüche dynamisch austrägt und sich dadurch verändert. Kritik ist, kurz gesagt, nicht mehr subjektiv, sondern objektiv. Es ist genau dieses Programm einer objektiven Kritik, das Marx im Kapital übernimmt. In einem Brief an Kugelmann schreibt er zur Methode des Kapitals:

Die Arbeit, um die es sich zunächst handelt, ist Kritik der ökonomischen Kategorien oder, if you like, das System der bürgerlichen Ökonomie kritisch dargestellt. Es ist zugleich Darstellung des Systems und durch die Darstellung Kritik desselben.“ (MEW 29, 550)

Bei Marx' Kapital handelt es sich, gewissermaßen analog zu Hegels Logik, um eine Darstellung des kapitalistischen Systems, das aus seiner Elementarform, der Ware, entwickelt wird, um aus innerer Konsequenz dieses Systems zu zeigen, wie es sich in Selbstwidersprüche und in Krisen manövriert. Die Kritik des Kapitalismus ist dabei keine Aufgabe mehr, die von einem spezifischen subjektiven Standpunkt an diesem System geübt wird, sondern dieses System kritisiert sich durch den Vollzug der eigenen Akkumulations- und Krisendynamik selbst. Diese objektive ,Selbstkritik“ führt schließlich geradezu von selbst in eine Selbstaufhebung. Für die bewusste politische Aktion von Subjekten bleibt in dieser Konzeption nicht mehr viel Raum. Wie Marx zum Ende des Kapitals schreibt, vollzieht sich diese Selbstaufhebung „durch das Spiel der immanenten Gesetze der kapitalistischen Produktion selbst [...]. [D]ie kapitalistische Produktion erzeugt mit der Notwendigkeit eines Naturprozesses ihre eigne Negation. Es ist Negation der Negation.“ (MEW 23, 790f.) Diese objektive Negation der Negation bezeichnet Marx als die geschichtliche Tendenz der kapitalistischen Akkumulation (vgl. MEW 23, 789). Die objektive Selbstkritik der kapitalistischen Akkumulation lässt sich daher als geschichtliche Kritik und das entsprechende Kritikmodell als geschichtsphilosophisch bezeichnen.

\section{Welche Kritik verändert die Welt?}

Rückblickend lassen sich den drei verschiedenen Kritikmodellen, der linkshegelianischen Kritik, der sozialrevolutionären Kritik und der geschichtsphilosophischen Kritik, drei verschiedene Verständnisse der gesellschaftlichen Wirklichkeit zuordnen: In seinen frühesten Schriften versteht Marx

\begin{tabular}{|l|l|l|l|l|}
\hline Q Ponista Dialectus & Ano 9 & n. 18 & Outubro 2020 & p. 202-218 \\
\hline
\end{tabular}


unter Kritik, dass Ideen bzw. Normen von subjektiven Akteuren als Maßstab an die falsche gesellschaftliche Wirklichkeit angelegt werden, um einen Widerspruch zwischen Normen und Wirklichkeit zu kritisieren. Kritik basiert in diesem linkshegelianischen Modell auf dem Widerspruch zwischen der objektiven Wirklichkeit und subjektiven Normen. Sowohl die Normen als auch die Kritik der Wirklichkeit auf Grundlage dieser Normen ist subjektiv. Bald darauf versteht Marx die Ideen bzw. Normen im Modell der sozialrevolutionären Kritik nicht mehr subjektiv, sondern interpretiert den Widerspruch zwischen Normen und faktischer Wirklichkeit als Widerspruch zwischen Staat und bürgerlicher Gesellschaft. Der Widerspruch zwischen Normativität und Faktizität ist somit keiner mehr zwischen der subjektiven Idee einerseits und der Gesellschaft andererseits, sondern der Widerspruch zwischen Normen und Faktizität wird selbst als gesellschaftlichinstitutioneller Widerspruch zwischen Staat und Markt, also objektiv interpretiert. Der Kritik fällt die Aufgabe zu, diesen Widerspruch innerhalb von gesellschaftlichen Konflikten zu explizieren und diese Konflikte an der radikalen Veränderung als Aufhebung dieses Widerspruches zu orientieren. Im Unterschied zum ersten Modell werden in diesem zweiten Modell die Normen objektiv verstanden, die Kritik ist aber nach wie vor subjektiv. Dies ändert sich im dritten Modell. Hier geht Marx in zweifacher Hinsicht über die ersten beiden Modelle hinaus, weil er Kritik erstens gar nicht mehr auf gesellschaftlichen Normen gründet und die Kritik zweitens nicht mehr subjektiv, sondern objektiv versteht: Der für die Kritik entscheidende Widerspruch ist nicht mehr der Widerspruch zwischen gesellschaftlich verstandenen Normen und der faktischen Wirklichkeit dieser Gesellschaft, sondern ein faktischer Selbstwiderspruch der Gesellschaft, nämlich der zwischen Kapital und Arbeit. Folglich ist die Kritik keine Aufgabe mehr von gesellschaftlichen Akteuren im Anschluss an gesellschaftliche Normen. Vielmehr vollzieht sie sich als gewissermaßen anonyme Selbstkritik objektiver Entwicklungstendenzen der kapitalistischen Akkumulationsund Krisenprozesse, die schließlich gleichsam automatisch zu ihrer Selbstaufhebung führen.

Während das erste linkshegelianische Modell einen letztlich unpolitischen Moralismus oder Voluntarismus begünstigt, droht das dritte geschichtsphilosophische Modell die kritische und praktische Initiative, die im sozialrevolutionären Modell im

\begin{tabular}{|l|l|l|l|l|}
\hline Q Povista Dialectus & Ano 9 & n. 18 & Outubro 2020 & p. 202-218 \\
\hline
\end{tabular}


Fokus steht, in einen geschichtsphilosophischen Determinismus aufzulösen. ${ }^{7}$ Obwohl das zweite sozialrevolutionäre Modell hier einen guten Kompromiss anbietet, bleibt in diesem Modell unklar, warum und wie sich aus der Diagnose objektiver gesellschaftlicher Widersprüche die subjektiv verstandene Kritik und Veränderung der Gesellschaft begründen lässt.

In Marx' Denkentwicklung lösen die Kritikmodelle einander ab und werden als Überbietung des überholten Vorgängermodells verstanden. Aufgrund der Stärken und Schwächen der jeweiligen Modelle scheint es aber aussichtsreicher, nicht eines dieser Modelle zu privilegieren, sondern sie jeweils als Elemente in einen umfangreichen Kritikbegriff zu integrieren: Die Kritik des Kapitalismus ist wohl kaum, wie es das dritte Modell nahelegt, allein durch eine Einsicht in objektive geschichtliche Krisentendenzen (die im Übrigen immer zweifelhafter werden, je länger der Kapitalismus fortbesteht) motiviert, sondern zumindest auch, wie im ersten Modell, durch subjektive Freiheits- und Gerechtigkeitsvorstellungen. Diese bleiben aber kraftlos, wenn sie als Normen im luftleeren Raum postuliert werden, sondern sie müssen sich, wofür das zweite Modell plädiert, in den gesellschaftlichen Kämpfen aktualisieren lassen, ihnen als brauchbarer Leitfaden dienen, und auch, wofür das dritte Modell steht, im Horizont der geschichtlichen Möglichkeiten formuliert werden. Erst dann lässt sich der Sozialismus jenseits von vermeintlichen harten Naturgesetzen der Geschichte einerseits und luftigen utopischen Hoffnungen andererseits als reale politische Möglichkeit begründen.

\section{Literatur}

MEW Marx, Karl/Engels, Friedrich: Werke. Hrsg. vom Institut für Marxismus-Leninismus beim ZK der SED. Berlin 1976ff.

Arndt, Andreas 2012: Karl Marx. Versuch über den Zusammenhang seiner Theorie. 2. Auflage. Berlin.

Hegel, Georg Wilhelm Friedrich 1830: Enzyklopädie der philosophischen Wissenschaften im Grundrisse. Erster Teil. 11. Auflage. Frankfurt am Main 2017.

7 Vgl. hierzu Iber 2018.

\begin{tabular}{|l|l|l|l|l|}
\hline Qovista Dialectus & Ano 9 & n. 18 & Outubro 2020 & p. 202-218 \\
\hline
\end{tabular}


- 1807: Phänomenologie des Geistes. Gesammelte Werke, Band 9. Hrsg. von Wolfgang Bonsiepen und Reinhard Heede. Hamburg 1980.

Heinrich, Michael 2014: Die Wissenschaft vom Wert. Die Marxsche Kritik der politischen Ökonomie zwischen wissenschaftlicher Revolution und klassischer Tradition. 6. Auflage. Münster.

- 2009: „Kritik bei Marx“. In: Devi Dumbadze u.a. (Hrsg.): Erkenntnis und Kritik. Zeitgenössische Positionen. Bielefeld, 41-48.

Iber, Christian 2018: „Revolution als Negation der Negation? Zu Marx” Hegelrezeption“. In: Allgemeine Zeitschrift für Philosophie 43.3, S. 249-266.

Röttgers, Kurt 1975: Kritik und Praxis. Zur Geschichte des Kritikbegriffs von Kant bis Marx. Berlin.

Spoo, Georg 2020: „Ideal. Polemik. Revolution. Drei Typen der Kritik beim frühen Karl Marx (1841-44)“. In: Doris Maja Krüger/Markus Börner (Hrsg.): Karl Marx in der Geschichte. Entstehung und Rezeption der Marx'schen Kritik. Bielefeld (im Erscheinen).

Strehle, Samuel 2019: „Karl Marx: Einleitung zur Kritik der Hegelschen Rechtsphilosophie (1844)“. In: Gärtner, Christel/Pickel, Gert (Hrsg.): Schlüsselwerke der Religionssoziologie. Wiesbaden, 45-55.

\begin{tabular}{|l|l|l|l|l|}
\hline Q Povista Dialectus & Ano 9 & n. 18 & Outubro 2020 & p. 202-218 \\
\hline
\end{tabular}

